\title{
A luminescence imaging system for the routine measurement of single-grain OSL dose distributions
}

Kook, Myung Ho; Lapp, Torben; Murray, Andrew; Thomsen, Kristina Jørkov; Jain, Mayank

Published in:

Radiation Measurements

Link to article, DOI:

10.1016/j.radmeas.2015.02.010

Publication date:

2015

Document Version

Peer reviewed version

Link back to DTU Orbit

Citation (APA):

Kook, M. H., Lapp, T., Murray, A., Thomsen, K. J., \& Jain, M. (2015). A luminescence imaging system for the routine measurement of single-grain OSL dose distributions. Radiation Measurements, 81, 171-177. https://doi.org/10.1016/j.radmeas.2015.02.010

\section{General rights}

Copyright and moral rights for the publications made accessible in the public portal are retained by the authors and/or other copyright owners and it is a condition of accessing publications that users recognise and abide by the legal requirements associated with these rights.

- Users may download and print one copy of any publication from the public portal for the purpose of private study or research.

- You may not further distribute the material or use it for any profit-making activity or commercial gain

- You may freely distribute the URL identifying the publication in the public portal 


\title{
A luminescence imaging system for the routine measurement of single-grain OSL dose distributions
}

\author{
M. Kook ${ }^{1 *}$, T. Lapp ${ }^{1}$, A.S. Murray ${ }^{2}$, K.J. Thomsen ${ }^{1}$, M. Jain ${ }^{1}$ \\ *Corresponding author: myko@dtu.dk \\ ${ }^{1}$ Center for Nuclear Technologies, Technical University of Denmark, DTU Risø Campus, Denmark \\ ${ }^{2}$ Nordic Laboratory for Luminescence Dating, Department of Geoscience, Aarhus University, Risø \\ Campus, DK-4000 Roskilde, Denmark
}

\begin{abstract}
In optically stimulated luminescence (OSL) dating and other retrospective dosimetry studies there is considerable demand for the ability to measure luminescence from individual dosimeters in the size range 50-500 $\mu \mathrm{m}$ diameter, either as separate grains or as part of a matrix. This work tests the potential of an electron multiplying charge-coupled device (EMCCD), providing extremely low level light detection. We characterize the performance of the device by discussing reproducibility and evaluating uncertainties in OSL signals. Finally we derive a typical single grain natural dose distribution with associated uncertainties.
\end{abstract}

Keywords: luminescence, imaging, single grain, EMCCD, dating 


\section{Introduction}

Luminescence is now widely used to date late Quaternary geological and archaeological sediments from many environments around the world. Unfortunately, many of these sediments (a) have not received sufficient light exposure to be fully reset, (b) have been mixed with other sediments after deposition, or (c) simply contain luminescence grains which do not act as reliable dosimeters. One solution to these problems is to examine dose distributions based on luminescence signals from individual grains (Duller et al, 1999, 2000).

In the past this has been achieved in routine application by stimulating each grain sequentially with a laser spot, and collecting the luminescence using a photomultiplier (PM) tube (Duller et al., 1999). An alternative approach is to stimulate all the grains simultaneously and image the resulting luminescence. Clark-Balzan and Schwenninger(2012) briefly summarized previous research into spatially resolved TL/OSL, and recorded UV OSL from natural quartz extracts through planoconvex lenses and a EMCCD (Qimaging, Rolera-MGi). Other recent work has also focused on the use of EMCCD devices, with imaging of TL/OSL of quartz and feldspar (Mundupuzhakal et al., 2014), and TL/OSL of quartz and radio-luminescence of K-feldspar (Richter et al., 2013). However, none of these instruments has been developed to the point where it can compete with the laser-based system in terms of automated analysis; this is an essential aspect of single-grain data collection, because of the very large number of luminescence signals that must be analysed to obtain sufficient data for a useful dose distribution.

In addition to the measurement of individually extracted and cleaned mineral grains, there is also an increasing demand for a system that can spatially resolve luminescence signals from solid samples, such as rock slices (e.g. Freiesleben et al., these proceedings) or biogenic carbonate (Duller et al., these proceedings).

In this paper, we first describe a new high sensitivity luminescence imaging system based on an EMCCD camera. The performance of the device is then characterised by discussing reproducibility and evaluating uncertainties in OSL signals. We specifically address the need to make repeat measurements of the same grain automatically, by developing a measurement strategy (involving both hardware and software) that allows individual measurements of many grains to be made without user intervention, i.e. without the need to manually define regions of interest for each grain 
after each measurement of an OSL image. Finally we illustrate results from this approach by deriving a typical single grain natural dose distribution with associated uncertainties.

\section{Instrumentation}

For the development of the new imaging system, we use a measurement platform consisting of a standard Risø TL-DA-20 reader fitted with a new 3 position OSL head with interchangeable filters and detectors (Lapp et al., these proceedings). The new camera unit is mounted on one of these detector positions.

\subsection{Optics}

The detection optics is based on $25 \mathrm{~mm}$ diameter fused silica lenses with anti-reflection coatings, providing high UV-visible transparency, large numerical aperture $(0.35)$ and magnification of $\sim 0.8$ (see Fig. 1). Object lenses collimate the light and projection lenses to produce an image on the sensor plane. One aspheric lens is used to enhance the image quality.

Because the location of the focal plane is dependent on the measured wavelength, the projection lenses of the optics are mounted on a motorized focusing unit, software-controlled by the measurement sequence. Before TL/OSL measurement, the location of the sensor plane is moved to the appropriate distance for the wavelength band to be measured (e.g. detection wavelengths for quartz and feldspar measurement are around $340 \mathrm{~nm}$ and $400 \mathrm{~nm}$, respectively). The motorized focusing unit is calibrated by three LEDs, emitting at $470 \mathrm{~nm}, 525 \mathrm{~nm}$ and $870 \mathrm{~nm}$. Sample thickness or location (e.g. disc, cup or rock slice) also affects the location of the focal plane.

As an alternative, near-UV achromatic optics (focal plane less sensitive to wavelength) is also available. This optics can be used for wide range of wavelength imaging without moving the position projection lens, but at the cost of a lower UV sensitivity. Only the performance of the fused-silica optics is investigated here.

\subsection{EMCCD}

Images are captured by a Peltier cooled $\left(-80^{\circ} \mathrm{C}\right)$ Evolve EMCCD camera (Photometrics); this uses frame transfer and electron multiplier gain (up to 1000 times). The imaging area $(512 \times 512,16 \mu \mathrm{m}$ 
pixel) of the chip is $8.2 \times 8.2 \mathrm{~mm}$, and so the object size is $10.2 \times 10.2 \mathrm{~mm}$ due to the reduction of the optics. The camera is connected to the host computer by IEEE-1394 (FireWire) for high-speed communication, and is triggered by a signal from the Risø controller for synchronization with stimulation light. Read-out time for all pixels is $29.5 \mathrm{~ms}$, but frame-transfer operation effectively reduces this considerably. In this mode, the image array is exposed to the signal for the desired period, and then the entire image is rapidly shifted $(0.7 \mu$ s vertical shift time) to the storage array. While the masked storage array is being read, the image array integrates charge for the next image, provides a minimum dead time of $\sim 400 \mu$ s between frames.

The EMCCD has a broadband (UV to Near IR, UV-enhanced) window and a high quantum efficiency (QE) compared to a PM tube (Fig. 2). However, a broadband QE has disadvantages when measuring OSL; the detector is more sensitive to the stimulation wavelength, and so more blocking of stimulation light is required. For instance, a U-340 filter is commonly used for quartz OSL measurement with a PM tube. But the U-340 filter has a small red transmission opening (around $715 \mathrm{~nm}$ ); the very weak red light emitted by blue LEDs is then visible to the EMCCD and this creates a large background count rate. To avoid this, a coated UV band pass filter (ITOS, UG11IRB UV-Broadband Filter) was used in our system. Black-body radiation in red and near-IR can also cause high background counts when measuring TL above $\sim 350{ }^{\circ} \mathrm{C}$.

There is one additional source of noise in an EMCCD that is not significant in a PM tube signal. A cosmic ray passing through the EMCCD device locally generates very high counts at a particular location in one frame. There is also a contribution to this effect from bremsstrahlung generated by the beta source. But all of these interferences only occur in one localised area, and in individual frames, with no corresponding signal at that location in adjacent frames; as a result they are readily identified and removed by e.g. median filtering of the image.

\section{Optics simulation and crosstalk}

\subsection{Sample holder}

The easiest approach to presenting grains for measurement is simply to spread them on a disc or a cup. But such loose grains can move from measurement to measurement, or even be lost from the sample altogether. Automatic OSL measurement then requires an algorithm and software to track 
the location of grains. In addition, when grains are spread randomly, some will lie very close to each other, or even touch. This increases crosstalk and the difficulty of repeated recognition of individual grains. Fixing the location of grains on a grid pattern helps to reduce or even eliminate such problems. We choose to use a standard single grain disc $(9.8 \mathrm{~mm}$ diameter aluminium disc with 100 grain holes; Duller et al., 1999) to present granular samples to the EMCCD camera. Each grain is contained within a $300 \mu \mathrm{m}$ deep by $300 \mu \mathrm{m}$ diameter hole, on $600 \mu \mathrm{m}$ grid spacing, and in addition there are 3 holes ( $500 \mu \mathrm{m}$ diameter) running completely through the thickness of the disc; these are used to locate the disc after each lift of the heater unit (Fig. 1).

\subsection{Optimization of detection efficiency and crosstalk}

One of the limitations common to all previous EMCCD-based instruments arises from the very weak signals emitted by individual quartz or feldspar grains. The very skewed distribution of sensitivities of quartz grains in particular (Duller et al., 2000) means that very few grains emit most of the light from an aliquot, and that even a small improvement in detection efficiency can give a significant increase in the number of usable grains. The crosstalk in single grain measurement based on laser stimulation is limited by the scattering of the stimulation laser beam; since the spot diameter is much smaller than the $300 \mu \mathrm{m}$ hole, crosstalk in a properly adjusted instrument is easily kept small (Duller, 2012). In an imaging system, crosstalk arises because of the inevitable overlap of grain images because of focussing limitations (e.g. spherical aberration, chromatic aberration, coma, astigmatism, etc.). This leads to a design conflict - on the one hand the numerical aperture should be maximised to collect as much light as possible, but on the other hand all grains are being observed at once; to record the signals from an insensitive grain adjacent to a bright grain, the optics must deliver a well-focussed image (using a reduced numerical aperture). So there is an unavoidable trade-off between detection efficiency and crosstalk. The spacing of our grains on a regular grid ensures a minimum separation of grain images, which reduces the crosstalk problem, but does not eliminate it.

Design optimization was carried out by simulation using the optics program ZEMAX (ZEMAX 12). As a source, we modelled a single grain disc with 100 uniform light sources (300 $\mu \mathrm{m}$ diameter, 600 $\mu \mathrm{m}$ grid spacing), and imaged it on the sensor through the optimised optics. The simulated line profile from one grain is shown in Fig. 3a (left axis), modelled using 500,000 simulated rays and a quartz UV-blue emission filtered through a U-340 (see Fig. 2). The experimentally observed 
luminescence profile from 10 grains of Risø calibration quartz (40 Gy beta dose, blue stimulation and $0.1 \mathrm{~s}$ integration) are also shown in Fig. $3 \mathrm{a}$ (right axis). The physical locations of these 10 grains (A to J) are shown in Fig. $3 \mathrm{c}$ as an optical image and in Fig. $3 \mathrm{~d}$ as a luminescence image. The average full-with half-maximum (FWHM) of the measured profiles is $273 \pm 10 \mu \mathrm{m}, \sim 17 \%$ wider that predicted by simulation $(234 \mu \mathrm{m})$; this small discrepancy is not surprising, because the source cannot be perfectly uniform, and the optics has inevitable manufacturing imperfections.

To quantify the crosstalk in this system, we consider the signal from an empty hole beside a bright grain. The 'empty hole' is imaginary; to ensure that the position does not contain a potentially light emitting grain, we examine a position on the appropriate grid spacing, but immediately outside the 10x10 grid (see Fig. 3c and Fig. 3d). The light sum from this imaginary hole, and from the adjacent hole (emitting light, because it contains a grain) is shown as a function of integration diameter in Fig. 3b. The solid blue line is the ratio of these two observations; if the signal is integrated over a $450 \mu \mathrm{m}$ diameter circle centred on a hole, then only $0.8 \%$ of the emitted light enters the adjacent integration area. However, this places considerable constraints on an automatic analytical system the centre of each hole must be accurately located, or this crosstalk will increase considerably.

\section{Typical measurement procedure}

\subsection{TL/OSL measurement procedure}

After every TL/OSL measurement the disc is almost always moved before it is measured again, because the heater lift lowers, and the carousel rotates. Thus we cannot rely on the grain holes being in exactly the same position from one measurement to another. To overcome this problem, a typical measurement consists of TL/OSL signal acquisition followed by the recording of an image under IR illumination. These luminescence and optical images are saved as TIFF files (Tag Image File Format) to ensure that all image information (counts per pixel) is recorded. The low power $(<0.08$ $\mathrm{mW})$ and short exposure time $(50 \mathrm{~ms})$ of the illumination light ensures no unintended stimulation of the sample. The wavelength of IR illumination $(870 \mathrm{~nm})$ is very far from measured luminescence wavelength (e.g. $340 \mathrm{~nm}$ or $400 \mathrm{~nm}$ ) and chromatic aberration leads to a defocussed image. Rather than adjust the focussing each time (time consuming) it is faster to move a small aperture (e.g. $1 \mathrm{~mm}$ ) into the optical path using the filter wheel. This improves the depth of field, and provides a wellfocussed image for further processing and hole location. 


\subsection{Image processing for single grain analysis}

An image-processing algorithm for detecting the 3 location holes in a single grain disc has been developed and implemented. It starts with information concerning possible location, expected hole diameter and shape and expected relative hole location to identify the centres of the location holes. Once these positions are found, the centres of all the grain holes are calculated and this information id used to control the spatial integration of the signal. The TL glow curves or stimulation decay curves from each grain are then calculated and saved to a conventional BIN file suitable for further analysis using ANALYST. Because all the image information is stored for each frame, the user is free to redefine integration intervals at any time after data collection, and recalculate the BIN file. The entire data extraction process is automatic; no user intervention is required during normal operation.

Such data processing is time consuming, but in practice, after each TL/OSL measurement, the reader moves on to the next measurement in the sequence. Simultaneously a separate thread program carries out the previous image processing while the next measurement is underway, so that when the sequence finishes there is a delay of only a few minutes before the BIN file is available for further analysis.

\section{Single grain TL/OSL}

It is well known that the intensity of the $110^{\circ} \mathrm{C}$ correlates with that of the OSL peak, and indeed the first version of SAR used the $110^{\circ} \mathrm{C}$ peak intensity to correct for sensitivity changes (Murray and Roberts, 1998). Murray and Wintle (2000) showed that because the $110^{\circ} \mathrm{C}$ peak was measured before the first OSL measurement, it could be used to monitor any changes in sensitivity that took place during the measurement of the natural. Singhvi et al. (2011) have tried to use this characteristic to monitor the sensitivity change across the measurement of the natural signal. Unfortunately, it has not been possible to implement such a correction at the single grain level, because the laser-based system does not permit the collection of TL signals from individual grains. Since the new luminescence imaging system can measure such single grain TL curves, we can now explore this correction at the single grain level.

To illustrate this advantage of the imaging approach, both TL and OSL measurement was undertaken using Risø calibration quartz (batch 90; Hansen et al., these proceedings). An optical 
(illuminated by IR) image and the first frame of an OSL image following a 5 Gy beta dose and TL to $220^{\circ} \mathrm{C}$ is shown in Fig. $4 \mathrm{a}$ and $4 \mathrm{~b}$, respectively. The TL signal was collected using a $5{ }^{\circ} \mathrm{C} / \mathrm{s}$ heating rate without a nitrogen purge, and with integration of the luminescence over $0.1 \mathrm{~s}$ collection intervals. The OSL image was collected with the sample held at $125^{\circ} \mathrm{C}$, and the frame integrates the first $0.1 \mathrm{~s}$ of stimulation. Fig. 4c shows the OSL curve for an individual grain (grain number 86), together with the TL glow curve collected during TL for the same sample. Fig. 4d plots the integral of the $110^{\circ} \mathrm{C}$ peak against the initial OSL intensity, for 26 repeated cycles of 20 Gy of beta dose, TL and OSL measurement. As expected there is a pronounced correlation between these two signals, and, for this grain at least the correlation passes through the origin, suggesting that the ratio of any TL to OSL pair would provide a useful sensitivity correction.

\section{Noise analysis of EMCCD}

Compared to a PM tube, an EMCCD camera has additional sources of noise; these include read-out noise, spurious noise from clock-induced charge, and amplification noise (Robbins and Hadwen, 2003).Thus one cannot simply predict the uncertainty on a photon count by assuming Poisson statistics, and using the square root of the count as a predictor of the standard deviation of the count, as is done in ANALYST.

The signal to noise ratio (SNR) for an EMCCD can be by (Liang et al. 2012):

$$
S N R=\frac{L_{*} t}{\sqrt{F^{2} L_{*} t+F^{2} p D_{*} t+F^{2} p C+\left(\frac{\sigma_{\text {read }}}{G}\right)^{2}}}
$$

Where $\mathrm{L}$ is the number of counts per unit time, and $\mathrm{t}$ is the integration time. $\mathrm{F}$ is the excess noise factor, D the number of dark current electrons per pixel; $\mathrm{p}$ the number of pixels; $\mathrm{C}$ the clock induced charge noise; $\sigma_{\text {read }}$ the read noise of the detector, and $G$ the EM gain.

The EMCCD sensor (serial number: A13L31000) is Peltier cooled to $-80^{\circ} \mathrm{C}$, providing a very low dark current (0.00013 electron/pixel/s), and read noise (44.3 electrons with $10 \mathrm{MHz}$ port and an analogue gain of 3.0) is rendered negligible by the electron multiplier (EM) gain. Clock induced charge noise is reduced by optimizing the parallel shift rate of the EMCCD. As a result, the major contribution to noise is the excess noise factor $(F)$ which is predicted to have a value close to $\sqrt{2}$. Thus, the variance $\left(\sigma^{2}\right)$ in the signal $\mathrm{N}$ is predicted to be $\mathrm{N} / 2$, rather than $\mathrm{N}$ as in a pure Poisson 
distribution. We estimate $\mathrm{F}$ by plotting the total variance against expected Poisson variance (i.e. total number of counts per pixel). The slope of this relationship gives a direct measurement of $\mathrm{F}^{2}$. The good agreement between the measured F (1.46) and the predicted F (1.41) is shown in Fig. 5. F is expected to be independent of count rate, down to and including background. For comparison a PMT will have a dark count rate of a few tens $\mathrm{s}^{-1}$, but the corresponding EMCCD signal from one grain location (collected over 400 pixels in $1 \mathrm{~s}$ ) will be close to zero. This is mainly because of the difference in detector area $\left(0.102 \mathrm{~mm}^{2}\right.$ in the EMCCD compared to $590 \mathrm{~mm}^{2}$ in a standard $50 \mathrm{~mm}$ diameter PM tube) used to collect the signal from a single grain. It is interesting to note that Adamiec et al. (2012) has carried out a similar study of PM tube behaviour, and shown that while many tubes have an F factor close to one (i.e. noise is close to Poisson) some high sensitivity tubes have an $\mathrm{F}$ factor $>1$. It appears that the F factor should be determined for both EMCCD and PM tubes, if accurate estimations of uncertainty are required.

\section{Reproducibility}

To determine the overall reproducibility of the imaging system, an aliquot of calibration quartz was given a dose of $7 \mathrm{~Gy}$, preheated to $260^{\circ} \mathrm{C}$ for $10 \mathrm{~s}$, and stimulated for $40 \mathrm{~s}$. This cycle was repeated 200 times and the systematic trend in the data arising from sensitivity change minimized by using alternate results as test dose in the L/T data shown Fig. 6a. The L/T data were then fitted with a trend line, and the relative standard deviation (RSD) around this line calculated (Fig. 6b). The mean RSD is $1.7 \% \pm 0.4 \%(n=82)$. This RSD arises from a combination of instrument reproducibility and signal noise; the latter should be a combination of a Poisson distribution and the excess noise factor F. The unfilled bar histogram (Fig. 6c) shows the effect of dividing all raw OSL data by 2 before processing; this is expected to leave only the instrument reproducibility and Poisson noise. Using these data, the instrument reproducibility is $-2.0 \pm 0.5 \%(\mathrm{n}=82)$. Clearly we have overestimated the effective value of $\mathrm{F}$. If we had divided the data by $1.7(\mathrm{~F}=1.30)$, the instrument reproducibility would be a more realistic 1\% (Fig. 6a, inset histogram). This inconsistency in the apparent value of $\mathrm{F}$ derived here and above is under investigation.

\section{Dose response curve and dose distribution}

To illustrate the application of the imaging system to routine single grain OSL measurement, we have used a SAR protocol (Murray and Wintle, 2000) on quartz extracted from a Namibian fluvial sand (Thomsen et al., 2007). The sample was measured using a preheat at $200^{\circ} \mathrm{C}$ for $10 \mathrm{~s}$, blue 
stimulation at $125{ }^{\circ} \mathrm{C}$ for $40 \mathrm{~s}$, and a cutheat to $200{ }^{\circ} \mathrm{C}$. All dose response curves consist of 4 regeneration doses, a recuperation point ( $0 \mathrm{~Gy})$ and repeat doses (recycling points). Fig. 7a shows a typical dose response curve passing through the origin and good recycling; typical natural and regeneration simulation curves are shown inset. Fig. $7 \mathrm{~b}$ shows the results from analysing 1200 such grains. Of these, $13 \%$ passed standard rejection criteria and were accepted into the dose distribution. For comparison, the expected dose of $411 \mathrm{mGy}$ is shown as a vertical red line; this dose is based on the OSL age of pottery within which the fluvial sand was trapped (Thomsen et al., 2007). The single grain data are clearly consistent with this expected dose.

It should also be noted that Duller et al. (these proceedings) have used this instrument to generate similar growth curves using TL images from solid irregular pieces of biogenic carbonate. They employ a similar image processing approach using an optical image of each piece to reproducibly position user-selected regions of interest on the TL images, and so generate integrated TL glow curves suitable for further processing.

\section{Conclusion}

We have developed a sensitive imaging system with which, for the first time, it is feasible to measure a dose distribution from a large number of grains. Optics has been developed to provide an appropriate compromise between high detection efficiency and low crosstalk; this compromise is helped by making use of the single grain disc (normally used for laser-based measurement) to hold grains in fixed positions. This in turn enables the accurate location of single grains using image processing - the key to making repeat measurements without user intervention. We have illustrated this by repeatedly measuring single grain TL and OSL signals from calibration quartz, and showing the expected correlation between these two signals. The data are analysed so that they can be read directly into standard analytical programmes such as ANALYST, and we have shown that by dividing the integrated counts by a factor of 2 , the uncertainty estimates built into ANALYST should be valid. However, instrument reproducibility measurements suggest that the standard F factor may be slightly overestimated. Finally we have shown that a natural OSL dose distribution from unheated quartz gives a result consistent with expectation, and others have used this instrument for the measurement of TL images and doses from biogenic carbonate. 


\section{References}

Adamiec, G., Heer, A. J., Bluszcz, A., 2012. A Statistics of count numbers from a photomultiplier tube and its implications for error estimation. Radiat. Meas. 47, 746-751.

Clark-Balzan, L., Schwenninger, J., 2012. First steps toward spatially resolved OSL dating with electron multiplying charge-coupled devices (EMCCDs): System design and image analysis. Radiat. Meas. 47 (9), 797-802.

Duller, G.A.T., Bøtter-Jensen, L., Murray, A.S., Truscott, A.J., 1999. Single grain laser luminescence (SGLL) measurements using a novel automated reader. Nucl. Instrum. Methods Phys. Res. Sect. B. 155 (4), 506-514

Duller, G.A.T., Bøtter-Jensen, L., Murray, A.S., 2000. Optical dating of single sand-sized grains of quartz: sources of variability. Radiat. Meas. 32, 453-457.

Duller, G.A.T., 2012. Cross-talk during single grain optically stimulated luminescence measurements of quartz and feldspar. Radiat. Meas. 47, 219-224.

Duller, G.A.T., Kook, M., Stirling, R. J., Roberts, H.M., Murray, A.S, (these proceedings), Spatially resolved thermoluminescence from snail opercula using an EMCCD, Radiat. Meas.

Freiesleben, T., Sohbati, R., Murray, A.S., Jain, M., Hvidt S., Jakobsen B., (these proceedings), Mathematical model quantifies multiple daylight exposure and burial events for rock surfaces using luminescence dating, Radiat. Meas.

Hansen, V., Murray, A.S., Buylaert, J.-P., Yeo, E.Y., Thomsen, K.J. (these proceedings), A new irradiated quartz for beta source calibration, Radiat. Meas.

Lapp, T., Kook, M., Murray, A.S, Thomsen, K.J., Buylaert, J.-P., Jain, M., (these proceedings), The new luminescence detection and stimulation head for the Risø TL/OSL reader, Radiat. Meas.

Liang, J.Y. and Li, D.G., 2012. Noise Analysis of EMCCD and Optimum Design of its Operating Mode. Advanced Materials Research 571, 229-233.

Mundupuzhakal, J., Adhyaru, P., Chauhan, N., Vaghela, H., Shah, M., Chakrabarty, B., Acharya, Y., 2014. FPGA based TL OSL system with EMCCD for luminescence studies. 2014 JINST 9 P04001.

Murray, A.S, Roberts, R.G., 1998. Measurement of the equivalent dose in quartz using a regenerative-dose single-aliquot protocol. Radiat. Meas. 29 (5), 503-515.

Murray, A.S., Wintle A.G., 2000. Luminescence dating of quartz using an improved single-aliquot regenerative-dose protocol. Radiat. Meas. 32, 57-73.

Richter, D., Richter A., Dornich K., 2013. Lexsyg - A new system for luminescence research. Geochronometria. 40 (4), 220-228.

Robbins, M. S., Hadwen, B. J., 2003. The noise performance of electron multiplying charge- 
coupled devices, IEEE transactions on electron devices 50 (5), 1227-1232.

Singhvi, A. K., Stokes, Stephen, C., Chauhan, N., Nagar, Y. C., Jaiswal, M. K., 2011. Changes in natural OSL sensitivity during single aliquot regeneration procedure and their implications for equivalent dose determination. Geochronometria 38(3), 231-241.

Thomsen, K.J., Murray, A.S., Bøtter-Jensen, L., Kinahan, J., 2007. Determination of burial dose in incompletely bleached fluvial samples using single grains of quartz. Radiat. Meas. 42 (3), 370-379. 


\section{Figure Captions}

Fig. 1. Schematic diagram showing the components of the luminescence imaging system mounted on a Risø reader.

Fig. 2 Comparison of EMCCD and PM tube response, LEDs emission, U340 (EMCCD: www.photometrics.com, PMT: www.electrontubes.com, LED: www.epitex.com, U-340: www.hoyaoptics.com). All curves should be read on the right hand axis except that of EMCCD and the photomultiplier tube, which are read on the quantum efficiency axis.

Fig. 3 (a) Line profiles of modelled source and simulated result for one grain (left axis), and line profiles of 10 measured grains (40 Gy of beta dose, blue stimulation and $0.1 \mathrm{~s}$ integration, right axis). (b) The number of counts as a function of integration diameter.(c) The optical image of single grain disc with quartz grains.(d) The OSL image of calibration quartz following a beta dose of 40 Gy and preheating at $220^{\circ} \mathrm{C}$ for $10 \mathrm{~s}$.

Fig. 4 (a) Optical image under low power IR illumination, with software- identified grain locations superimposed. (b) OSL image of calibration quartz following a beta dose of 5 Gy and TL to $220^{\circ} \mathrm{C}$ for 10 s. (c) TL and OSL curves for grain no. 86. (d) Correlation between TL and OSL.

Fig. 5 Variance plotted against incident photon rate.

Fig. 6 (a) Reproducibility of the imaging system. The inset shows distribution of $L_{x} / T_{x}$ signals after correcting using $F=1.30$. (b) The distribution of observed RSD ( $\mathrm{R}_{\text {lin fit }}$ ) for uncorrected signals. (c) The distribution of RSD observed after dividing all raw OSL data by 2 .

Fig. 7 (a) The dose response curve from quartz extracted from a Namibian fluvial sand. The inset shows natural and regenerated OSL signals. (b) Single grain dose distribution. The single grain data are clearly consistent with the expected dose $(411 \mathrm{mGy})$. The inset shows the same data as a histogram. 


\section{Figure 1}

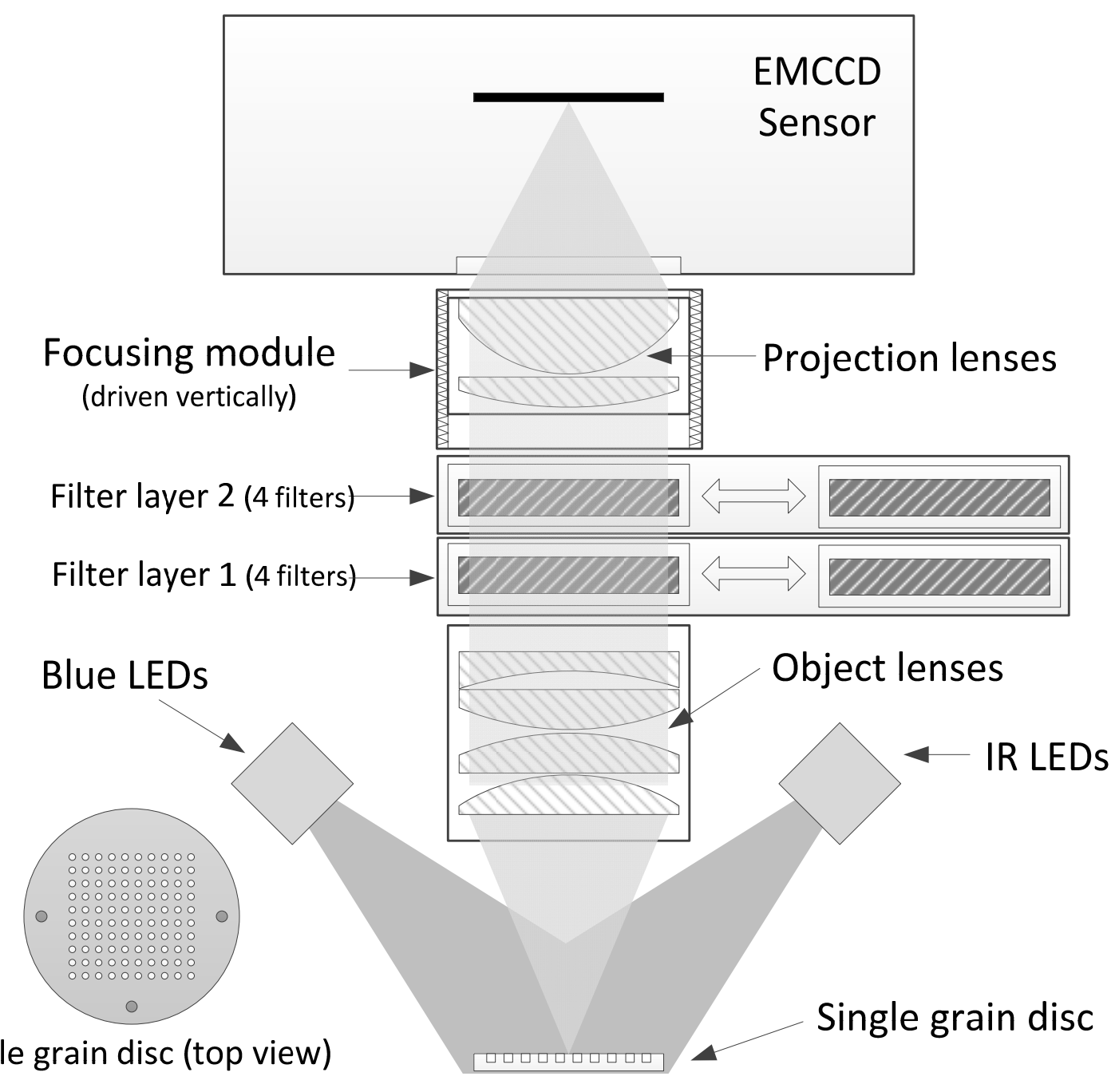

Single grain disc (top view) 
Figure 2

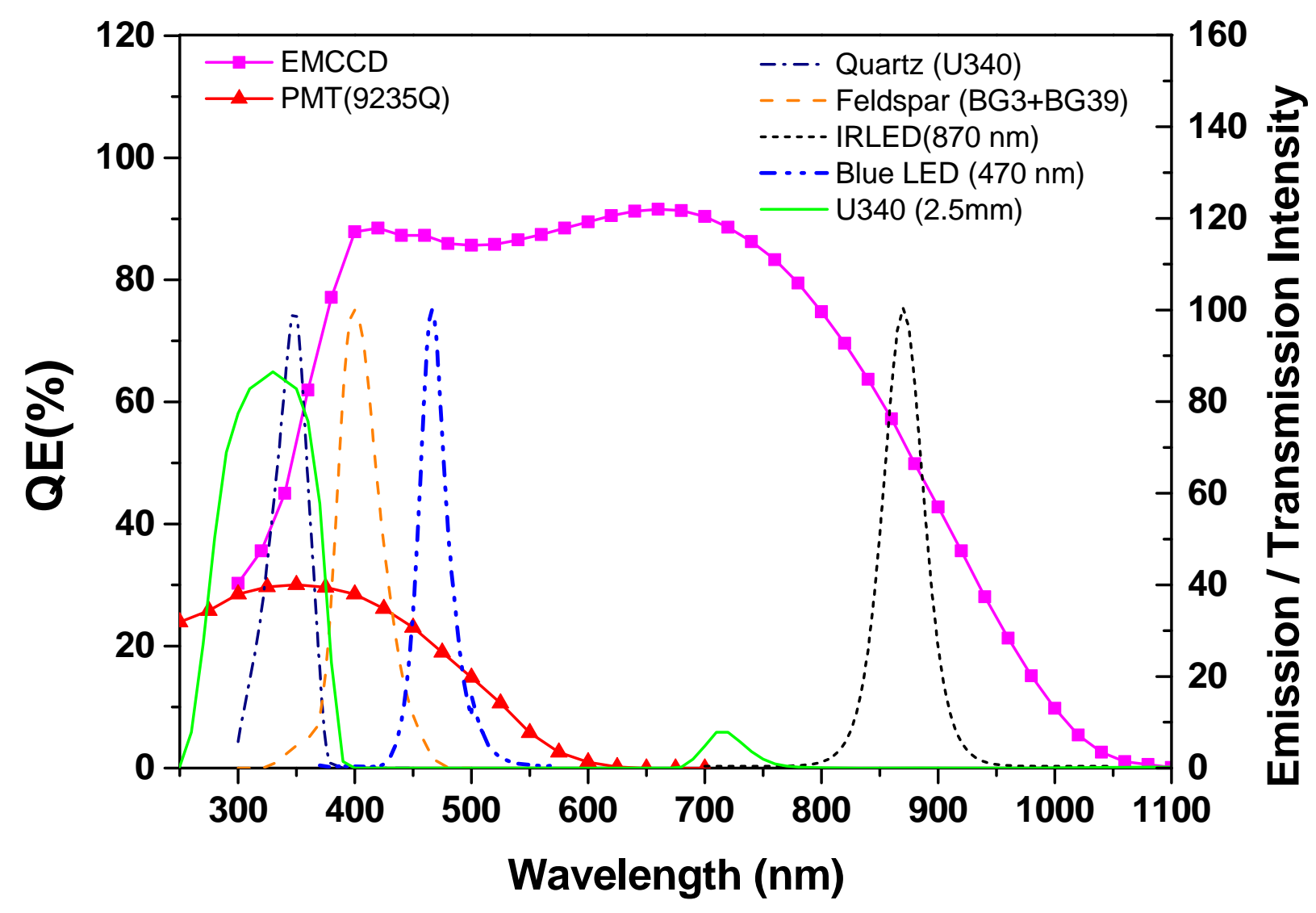




\section{Figure 3}

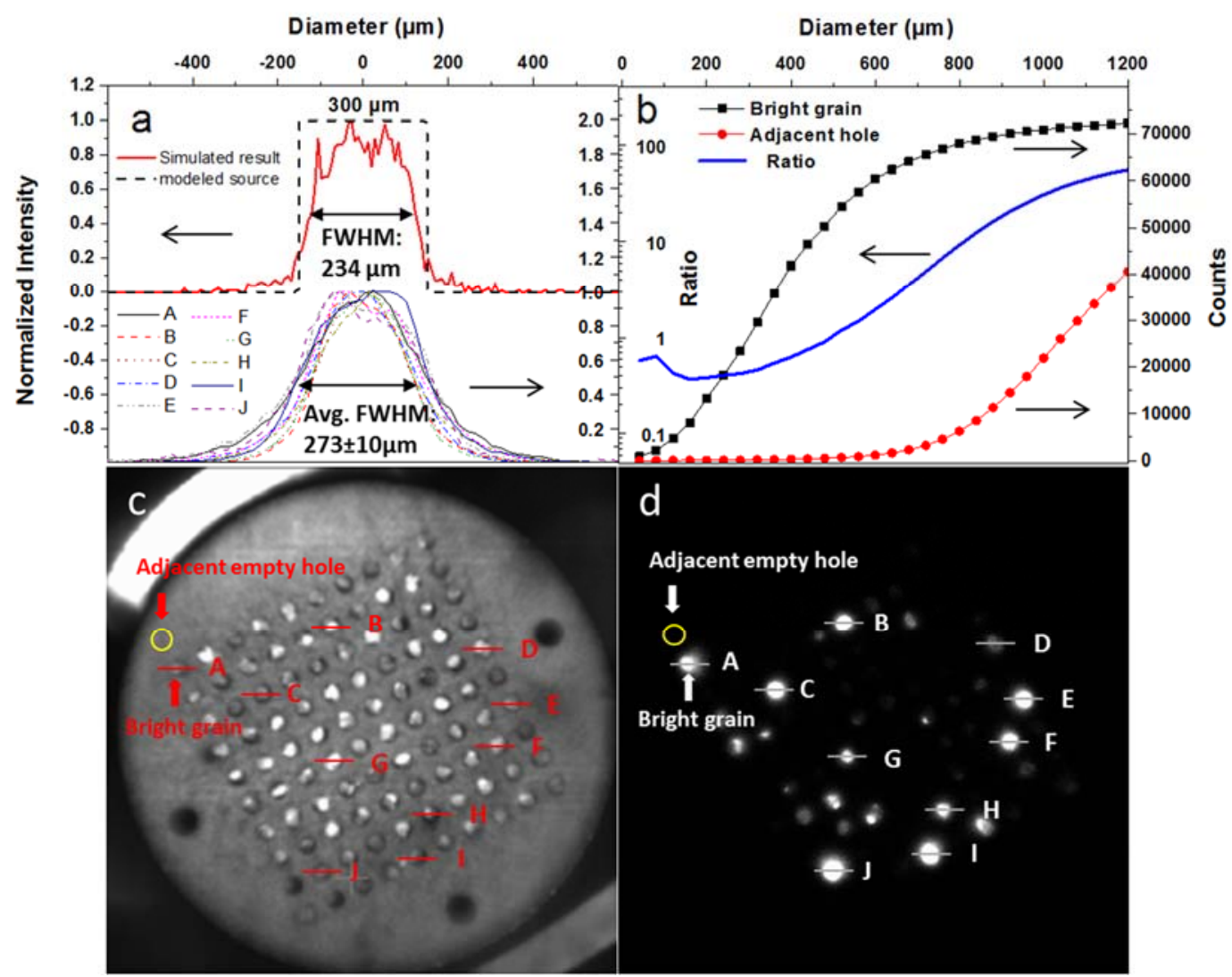


Figure 4

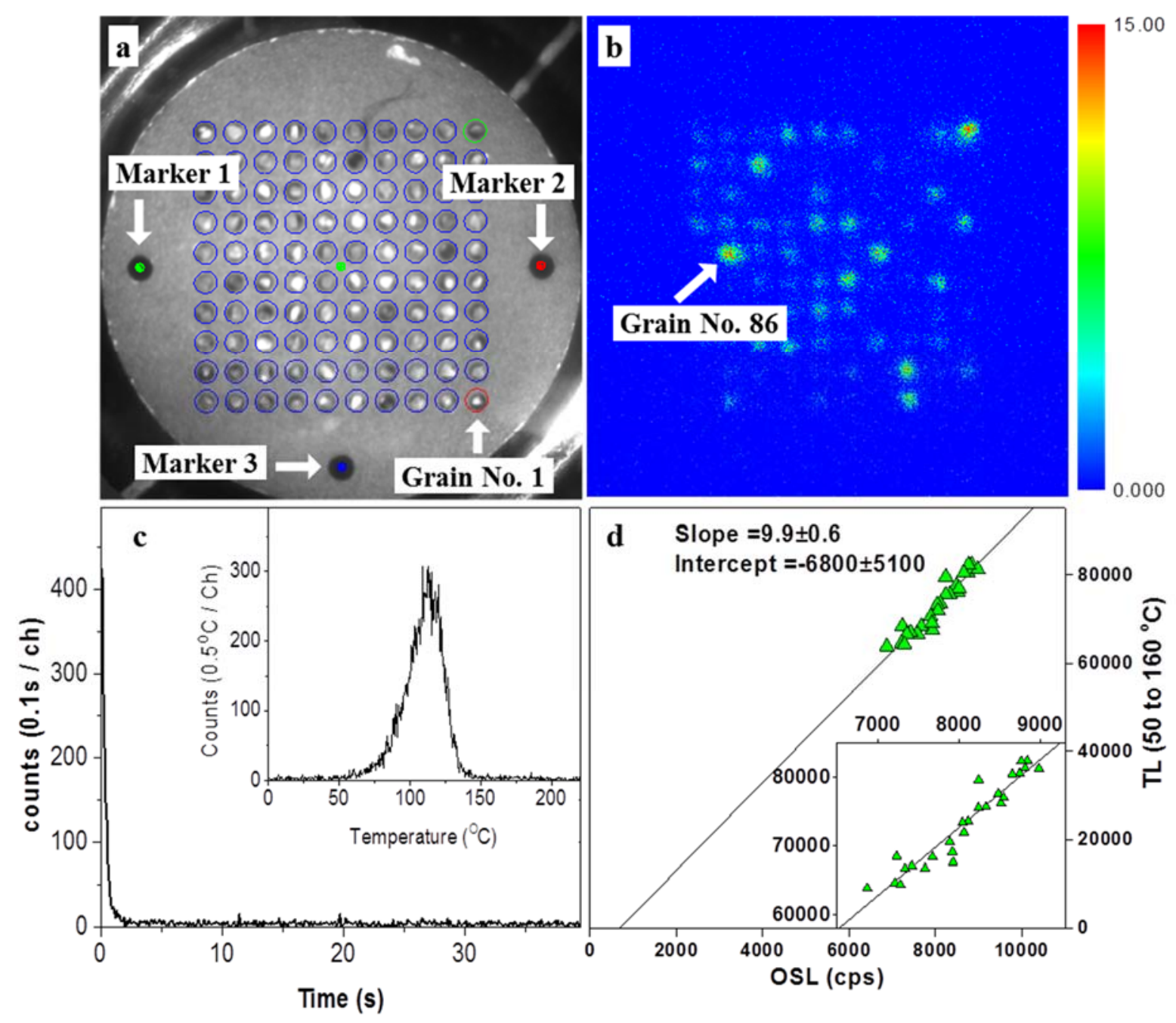


Figure 5

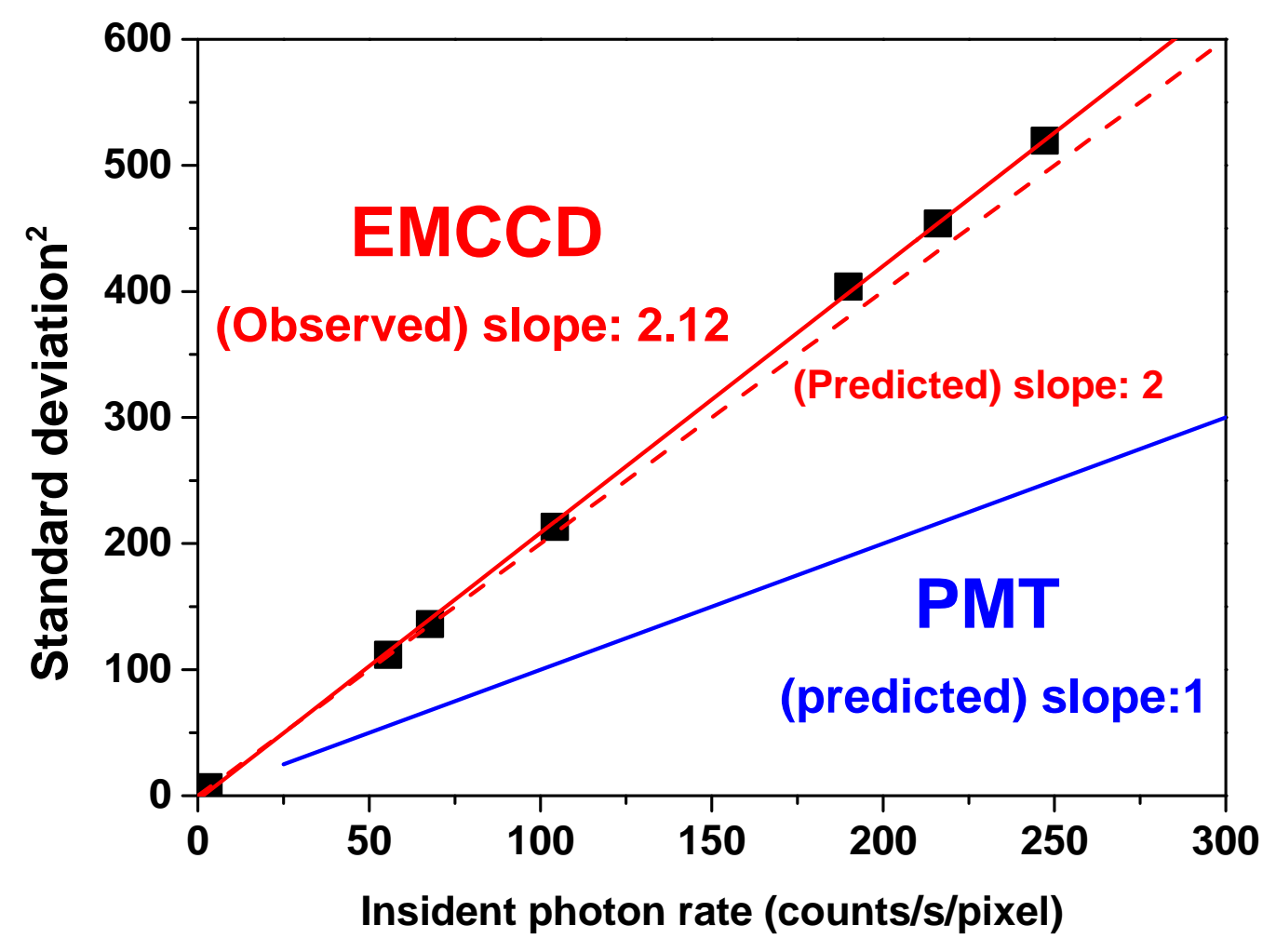


Figure 6
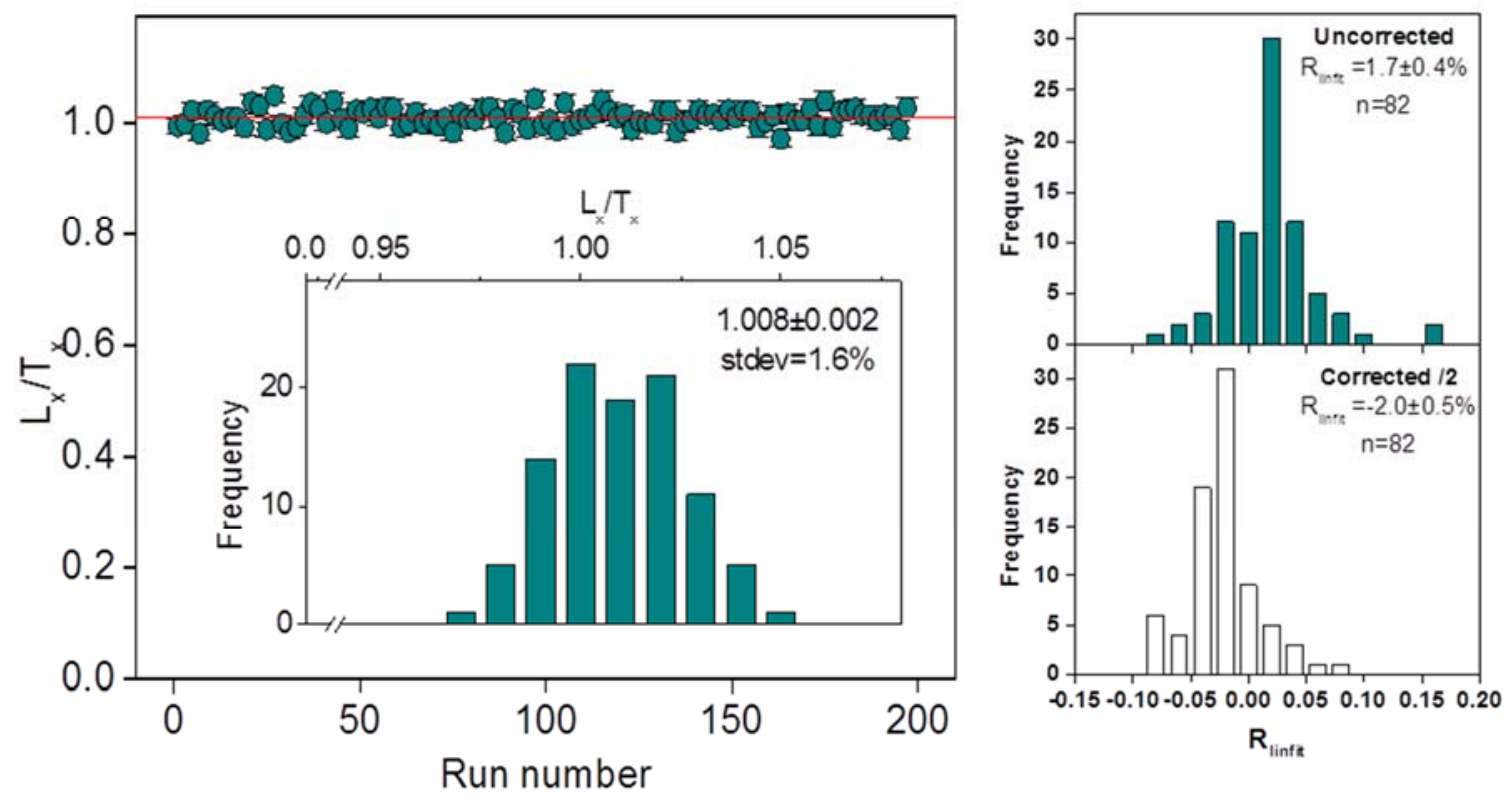
Figure 7

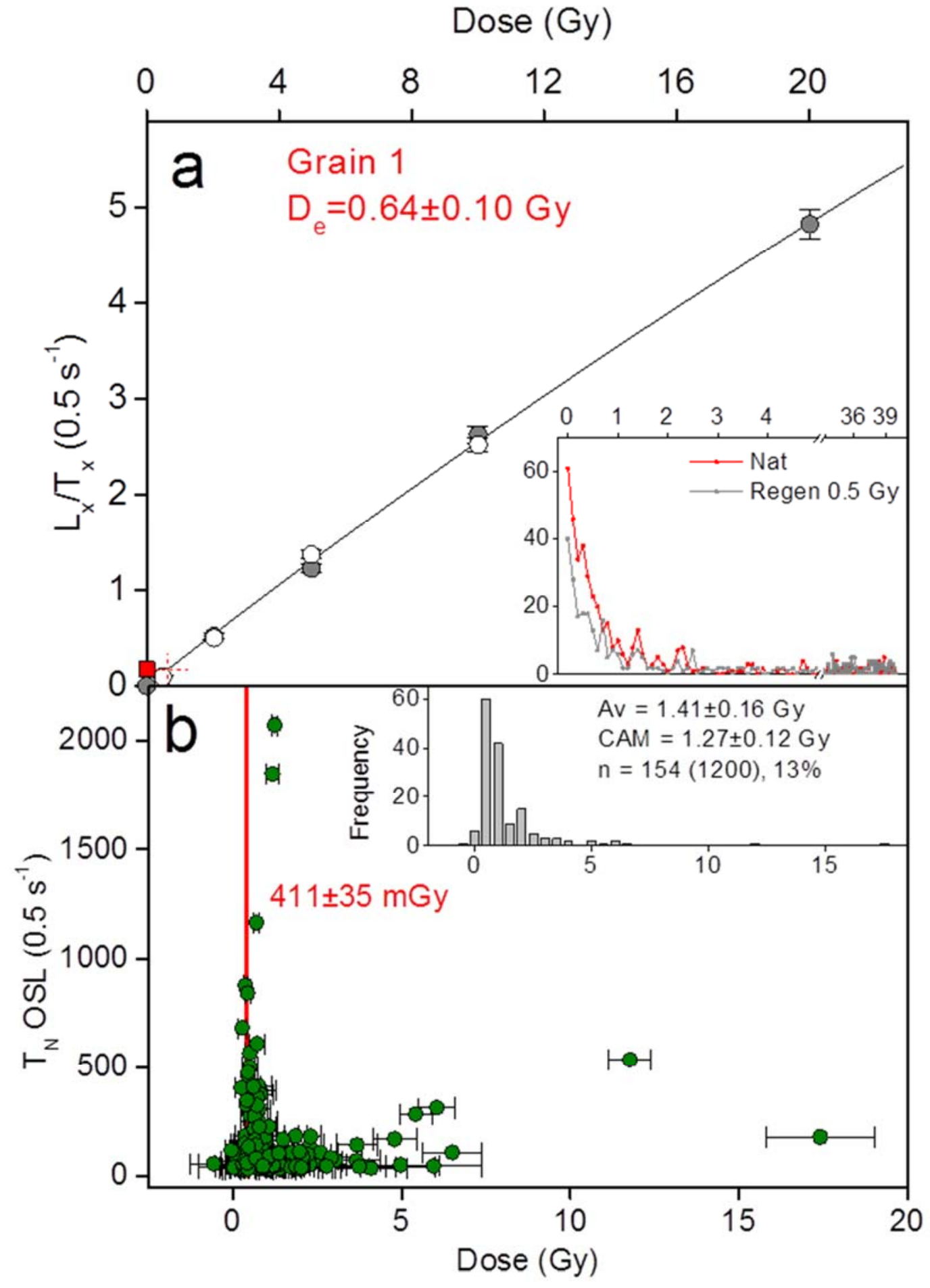

